\title{
Selected thermal and flow issues in a reversed thermosiphon with a steam liquid lifter
}

\author{
Michał Klugmann ${ }^{1, *}$, Paweł Dąbrowski ${ }^{1}$, and Dariusz Mikielewicz ${ }^{1}$ \\ ${ }^{1}$ Gdańsk University of Technology, Faculty of Mechanical Engineering, Department of Energy and Industrial Apparatus, Narutowicza \\ 11/12, 80-233 Gdańsk, Poland
}

\begin{abstract}
The work presents initial studies of a reversed thermosiphon with a steam liquid lifter and a minigap evaporator. The main interest of the authors is the evaporator, especially the heat exchange efficiency and flow issues, such as maldistribution and pressure drop. However, because the thermosiphon circuit of such a construction is characterized by cyclic working conditions, which are quite difficult in analysis the authors focused on this specificity first. The article presents the concept of a test circuit and preliminary results for distilled water and for a minigap evaporator with a smooth heat exchange surface $100 \times 200 \mathrm{~mm}$ and minigap thickness of $1 \mathrm{~mm}$. Visualizations of the separator's work and the minigap boiling structures are presented.
\end{abstract}

\section{Introduction}

All technology sectors are endeavouring to miniaturization with simultaneous performance improvement. That causes an increase of heat flux which is necessary to dissipate from the surface. For example, the average value of heat flux for computer processors is from 2 to $4.5 \mathrm{MW} / \mathrm{m}^{2}$ (up to $45 \mathrm{MW} / \mathrm{m}^{2}$ in small areas); for insulated gate bipolar transistors (IGBT) - from 6.5 to $50 \mathrm{MW} / \mathrm{m}^{2}$. Hence, there is a need to focus on modern kinds of highly compacted and high efficiency heat exchangers design and to maximize the heat transfer. Over the past decades, many scientists have focused on mini and microchannel geometries. It have been well described in the literature. There were also concepts to apply such geometries to the construction of highly efficient, compact heat exchangers. Much less attention was paid to the minigaps. Meanwhile, the minigap geometry seems to be - in some cases - even more interesting because of technically easier construction and very high heat transfer coefficients which have already been observed.

In addition, in recent times, the renaissance of all types of passive heat and mass transfer techniques has been observed. For example, in the water supply technique the gravitational systems based on the natural differences in terrain levels return to the favours, which were commonly used at the turn of the 19th and 20th century. Noticeable attention is paid to passive methods of heat exchange intensification. Thermosiphon circuits, a few decades ago widely used in heating systems, now focus the researchers' attention again. However, these are not only just simple systems that use single-phase convective flow from the lower to the upper heat source but also reversed systems, giving the heat flow from top to bottom, using two-phase flows and microscale geometries. This results in completely new research issues, both in the field of basic research as well as operational problems. One of such issues is cyclic/pulsatile work characteristic for some designs of reversed thermosiphons. This is a specific type of working conditions, from the borderline of a steady and transient state, which has rarely been investigated by researchers yet. One of the issues that takes on a key meaning in this type of work is a maldistribution phenomenon which can occur wherever there is a necessity of the working medium distribution within a complex flow system [1]. Examples of such systems may be minichannel and minigap plate heat exchangers.

The aim of the present paper is the research of reversed thermosiphon with a steam liquid lifter regarding the way the system works and the impact of basic parameters on its operation. The working fluid is distilled water operating under sub-atmospheric pressures.

\section{State of the art}

Different aspects of two-phase thermosiphons performance have been investigated over the years. Filippeschi [2] analysed Closed Loop Two Phase Thermosiphon (CLTPT). The purpose of this study was to find a correlation between mass flow rate and heat flow rate. Authors have been running experiments with water and ethanol as a working fluid, where heat flow rate was up to $1.7 \mathrm{~kW}$. The mass flow rate were measured at three different modes: a continuous mode, an integral mode and an indirect mode. They reported that the quantitative prediction of the performance of CLTPT appears to be quite difficult due to the individual

Corresponding author: garyb@pg.gda.pl 
and the inter-correlative effects of the various variables(operating pressure, filling ratio) but the maximum mass flow rate always can be found with the increase of the heat load.

Buschmann and Franzke [3] conducted experiment on vertical two-phase thermosiphon utilizing deionized water and water based nanofluids with titanium dioxide and gold particles in different concentrations. Their results show a decrease of the thermal resistance with increasing concentration of nanoparticles. Authors achieved a maximal thermal resistance reduction of about $24 \%$.

The employment of nanoparticles in thermosiphon performance was also an aim of study conducted by Cieśliński [4]. The results obtained with a nanofluid water-A12O3 solution as a working fluid were compared with a distilled water. Study showed that addition of $\mathrm{A} 12 \mathrm{O} 3$ nanoparticles results in a higher overall heat transfer coefficient but increase in nanoparticle concentration from $0.01 \%$ to $0.1 \%$ causes a decrease of the overall heat transfer coefficient.

The air to air two phase closed thermosiphon based heat exchangers were investigated experimentally by Danielewicz et al. [5]. Authors found out that effectiveness of heat exchanger increased as the ratio between both massflow rates increased. They validated the computational model that was used to predict the heat transfer coefficient, effectiveness, pressure drop and heat extraction duty of featured heat exchanger.

Zhu and Hrnjak [6] presented experimental evaluation of a two-phase thermosiphon loop in two modes: conventional and with separation. Microchannel heat exchanger was used as an evaporator. Authors measured temperature distribution over the evaporator surface in time. It seems that thermosiphon with separation of vapor and liquid phase stabilizes in shorter time than conventional one. Moreover separation causes lower mass flow rate oscillations at start-up. Besides, at low heat load in steady state operation, the total pressure drop in two phase line of the system with separation is lower than that of the system without separation.

Another utilization of microchannels in passive, gravitational systems has been realized by Panse and Kandlikar [7]. They used Open Microchannel Manifold (OMM) with taper configuration as an evaporator in thermosiphon loop. Ethanol was used as a working fluid. Results shown that OMM provides superior heat transfer while maintaining a low pressure drop performance. Authors prepared the boiling curves for $4 \%$ taper and $6 \%$ taper manifolds. A maximum heat flux of $136 \mathrm{~W} / \mathrm{cm}^{2}$ was recorded at a wall superheat of $42^{\circ} \mathrm{C}$ resulting in a highest heat transfer coefficient of $34.1 \mathrm{~kW} /\left(\mathrm{m}^{2} \mathrm{~K}\right)$ with $6 \%$ taper manifold.

Khodabandeh and Furberg [8] conducted research on single rectangular microchannel working as an evaporator in a two-phase thermosiphon loop. Authors enhanced the heat transfer surface of the evaporator with a copper nano- and micro-porous structure. Then the heat transfer of the enhanced evaporator was compared to a smooth surface evaporator. The main observation was that the enhanced structure surface decreased the oscillations at the entire range of heat fluxes and enhanced the heat transfer coefficient.

Bieliński and Mikielewicz [9] developed a onedimensional separated two-phase flow and heat transfer model in a closed thermosyphon loop with minichannels and a minipump. Studies indicated that the presented model is a power tool for increasing the effectiveness of computer cooling. If the mass flow rate is not high enough to circulate the necessary fluid to transport heat from evaporator to condenser, the minipump can be used to promote natural circulation. Another paper by Bieliński [10] validated mentioned above model by experimental measurements of volumetric flow rate. Author claimed that the steady-state conditions played a dominant role during the two-phase flow in the thermosiphon loop.

As shown above, there are some works that are trying to develop a microchannel geometries in a gravity-driven loops, i.e. thermosiphons. But there are hardly any research that conducts experiment on a microgap heat exchanger in application to passive systems (thermosiphons or heat pipes). However, there are some works on microgaps that are not necessarily related to passive systems but can be useful.

Nishikawara and Nagano [11] developed a mathematical model to investigate the heat transfer characteristics of a capillary evaporator in a loop heat pipe with a microgap between the case and the wick. Calculations were compared with measured results obtained for a polytetrafluoroethyleneethanol loop heat pipe. Authors found out that the heat-transfer coefficient have a local maximum against the gap distance.

Tamanna and Lee [12] dealt with various configurations of microgaps with expanding depth towards the outlet. A microgap with a constant depth of $200 \mu \mathrm{m}$ was used as a reference one. The gaps with increasing depth had the same initial depth of $200 \mu \mathrm{m}$ (inlet) and the final depth of $300 \mu \mathrm{m}$ and $460 \mu \mathrm{m}$ (outlet). Water with different mass fluxes from $G=400 \mathrm{~kg} / \mathrm{m}^{2} \mathrm{~s}$ to $\mathrm{G}=1000 \mathrm{~kg} / \mathrm{m}^{2} \mathrm{~s}$ was used as the working fluid. A variable heat flux of 0 $\mathrm{W} / \mathrm{cm}^{2} \div 80 \mathrm{~W} / \mathrm{cm}^{2}$ was supplied to water. Authors claimed that inlet pressure oscillations increase with increasing mass flow rate for a specific heat flux. This oscillations also increase with increasing heat flux for a predetermined mass flow rate. Pressure oscillations at the inlet to the exchanger can be lowered, using microgaps with expanding depth from $200 \mu \mathrm{m}$ to 300 $\mu \mathrm{m}$. However, it should be emphasized that further increasing of depth to $460 \mu \mathrm{m}$ does not give positive results. A similar situation occurs when it comes to temperature fluctuations.

Alam et al. [13] carried out tests to determine the heat transfer coefficient. They used microgaps at three different depths: $190 \mu \mathrm{m}, 285 \mu \mathrm{m}$ and $381 \mu \mathrm{m}$, through which water ran at an inlet temperature of $86^{\circ} \mathrm{C}$. The influence of the mass flow rate and the heat flux supplied to the heat exchanger on heat transfer coefficient and pressure drops was investigated. They found out that smaller the size of the microgap, the higher the local heat transfer coefficient. In addition, reducing the mass flow of the flowing medium also results in an increase in the heat transfer coefficient. 
Later Alam, Lee, and Yap [14] decided to carry out research on the same experimental rig as before, but this time they put the main emphasis on the surface roughness of microgaps. Flow visualization showed that the surface roughness has a significant impact on the formation of bubbles. The bubble nucleation density increases with increasing roughness. It is worth mentioning that this effect is greater for larger gap depths. The most important positive effect that can be achieved by using rough surfaces is to improve the heat transfer coefficient. The authors did not observe increased pressure drops when using rough surfaces. However, higher roughness results in increased fluctuations in pressure at the inlet to the heat exchanger.

As can be seen from the literature review, thermosiphons are very promising heat transfer devices. The lack of external source of working fluid circulation results in higher system efficiency and better reliability. There are some applications of passive methods with microchannels that provides high performance and miniaturization. Unfortunately, there is hardly any study that combines minigaps or microgaps in passive systems, and those structures ensures very good performances of heat transport. Current study aims to fill this gap in literature. Authors built the specially designed apparatus of reversed thermosiphon with minigap evaporator. The evaporator is above the condenser, which is justified in solar applications.

\section{Experimental setup}

Experimental rig, Fig.1., like any inverted thermosiphon has an evaporator located above the condenser. In the discussed system, this height difference, and thus, the lifting height is about $1.35 \mathrm{~m}$. The evaporator (4) is a plate heat exchanger composed of a single smooth plate with a heat exchange surface of $100 \times 200 \mathrm{~mm}$. Between the plate and the cover of the exchanger, a $1 \mathrm{~mm}$ thick minigap is arranged.

The heat for the evaporator can be supplied in two ways: a) radiative, through a transparent cover, similar to a solar collector; b) using a water circuit, where the temperature and flow of the water can by regulated in the thermostat. The assumption is to obtain the boiling structures in the minigap which can push the liquid upward into the separator (5) located above the evaporator. It is a liquid transport mechanism similar to those found in the mammoth pump.

In the separator, a liquid part of the working medium flows to the condenser through a pipeline (2) in the bottom of the separator, while the vapour part goes to the pipeline (3) at the top of the separator and further goes to the condenser through the second tube.

The condenser (6) is a coil immersed in a thermostat which allows precise temperature control and, thereby, the amount of heat removed from the system.

In steady state with an equal thermal balance, the system works in a characteristic cyclical way, throwing the portions of liquid and vapour into the separator at regular time intervals.

Evaporator and separator are equipped with sight-glasses
To observe and register the minigap boiling structures and the way of the separators work.

The system is equipped with temperature sensors at locations of interest and also at additional points on the length of pipelines to accurately analyze the temperature distribution in the entire circuit. The pressure difference is measured at the inlet and outlet of the evaporator. In addition, system pressure is measured.

The flowrate of the working medium is measured at the inlet to the evaporator (7) and at the outlet of the liquid pipeline from the separator (8). The flow rate in the vapour pipeline must be estimated from the mass balance.

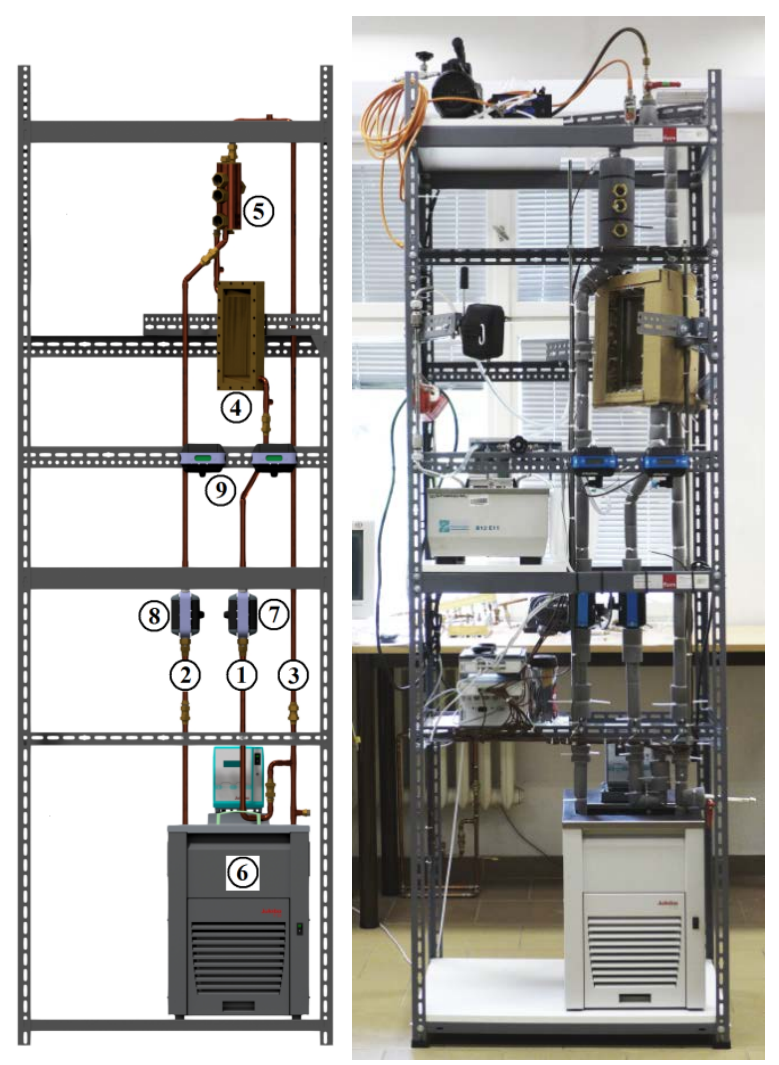

Fig. 1. Diagram and picture of experimental rig: 1- cold (input) pipeline, 2 - hot (output) liquid pipeline, 3 - hot (output) vapour pipeline, 4 - minigap evaporator, 5 - separator, 6 condenser, 7 - flowmeter for cold pipeline, 8 - flowmeter for hot liquid pipeline, 9 - display.

\section{Experimental results}

\subsection{System operation over the time}

The following is a comparison of the way the system works when supplying heat by means of a water circuit at two different mass flow rates. This results in an approximately twofold difference in the heat flux density supplied in the evaporator to the working medium. Registrations were carried out for the time of 100 seconds. 

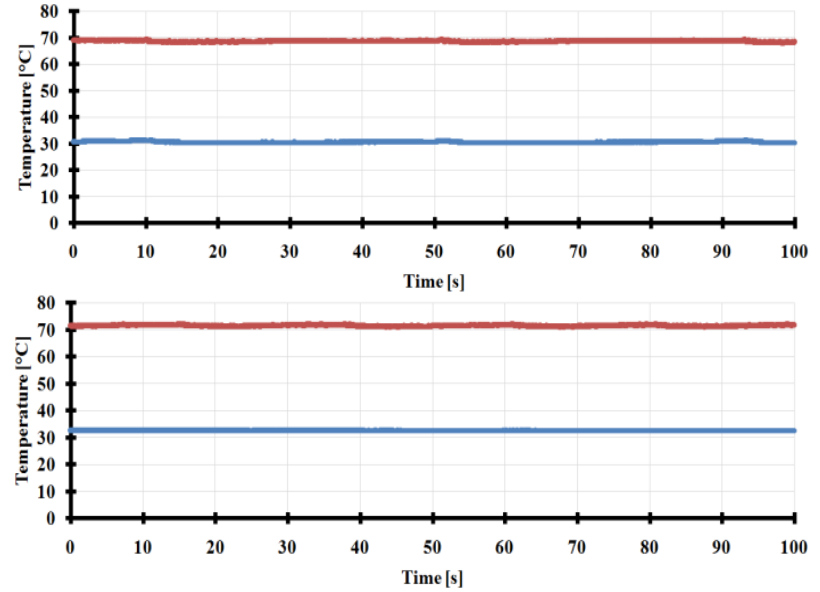

Fig. 2. Temperatures in evaporator (blue - input, red - output) for average heat flux supplied to minigap: top $=7.6 \mathrm{~kW} / \mathrm{m}^{2}$, bottom $=14 \mathrm{~kW} / \mathrm{m}^{2}$.
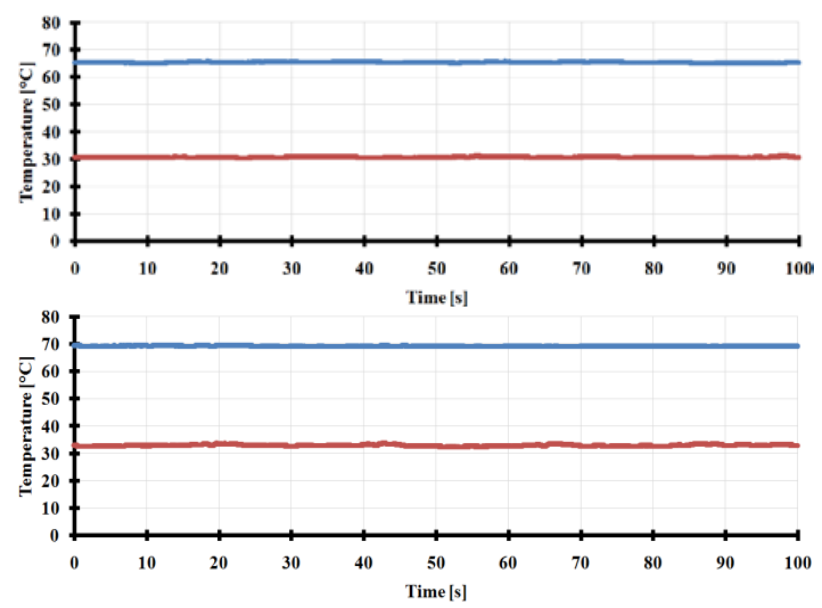

Fig. 3. Temperatures in condenser (blue - input, red - output) for average heat flux supplied to minigap: top $=7.6 \mathrm{~kW} / \mathrm{m}^{2}$, bottom $=14 \mathrm{~kW} / \mathrm{m}^{2}$.

Equal temperature distributions in time in the evaporator and condenser, as well as the fact that the working fluid is being cooled in the condenser to its initial temperature prove that the system, in both cases, works in even heat balance conditions and attains the equilibrium conditions.
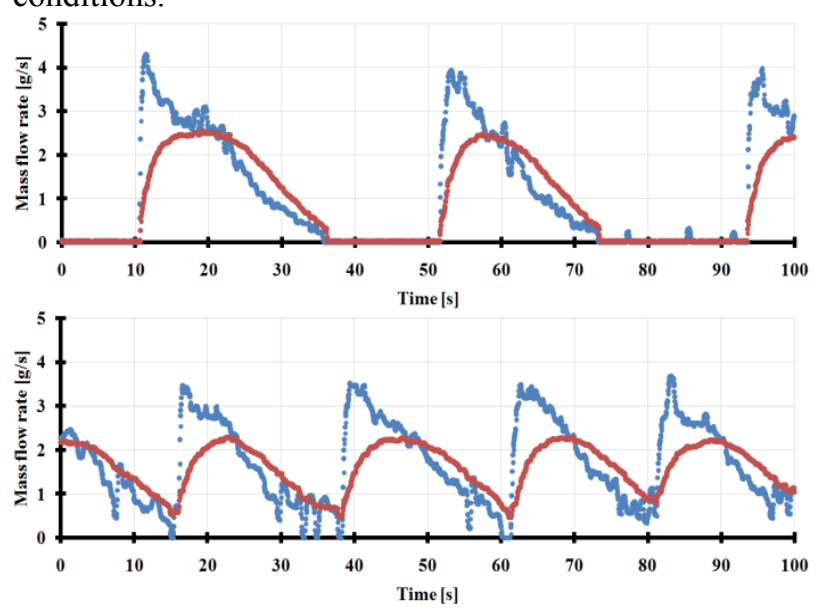

Fig. 4. Mass flowrate: blue - at evaporator input, red - at separator output (liquid pipeline) for average heat flux supplied to minigap: top $=7.6 \mathrm{~kW} / \mathrm{m}^{2}$, bottom $=14 \mathrm{~kW} / \mathrm{m}^{2}$.
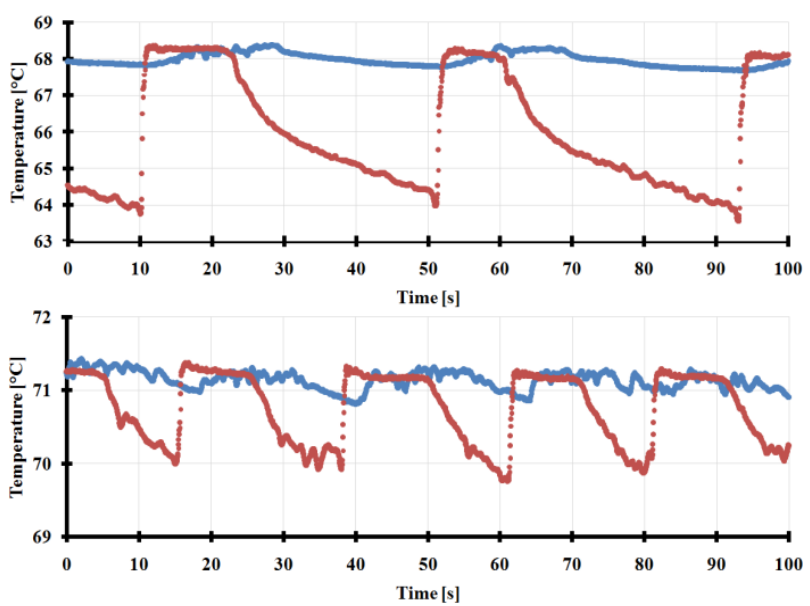

Fig. 5. Temperatures on separator's ouputs: blue - liquid pipeline, red - vapour pipeline for average heat flux supplied to minigap: top $=7.6 \mathrm{~kW} / \mathrm{m}^{2}$, bottom $=14 \mathrm{~kW} / \mathrm{m}^{2}$.
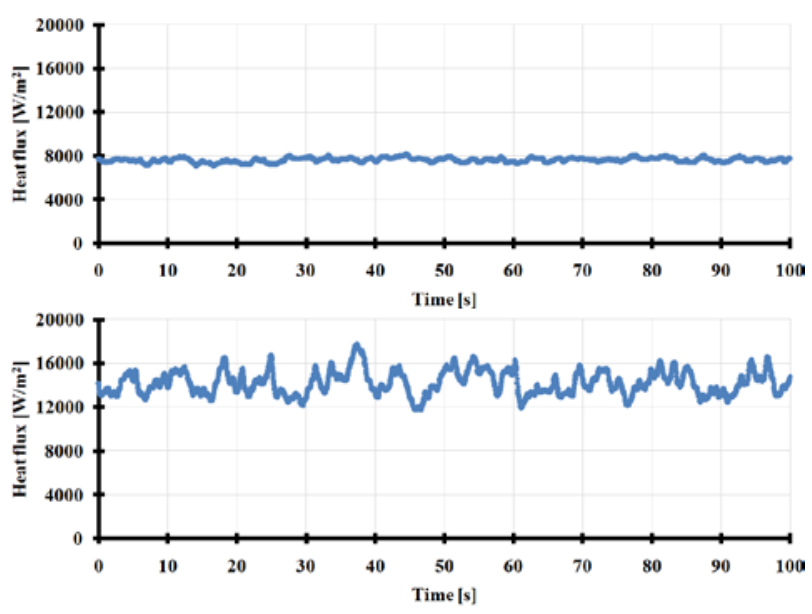

Fig. 6. Heat flux supplied to minigap: top $=7.6 \mathrm{~kW} / \mathrm{m}^{2}$, bottom $=14 \mathrm{~kW} / \mathrm{m}^{2}$.

In the mass flow rate charts there appears characteristic pulsating nature of the system operation. It can be seen, that for almost twice heat flux delivered the almost twice density of work cycles occurs.

Considering that - in both cases - the temperature differences in exchangers are of the same order of magnitude, we can conclude about how the system increases the amount of transferred heat. This is confirmed by the Fig. 7. where more heat flux density points are included.

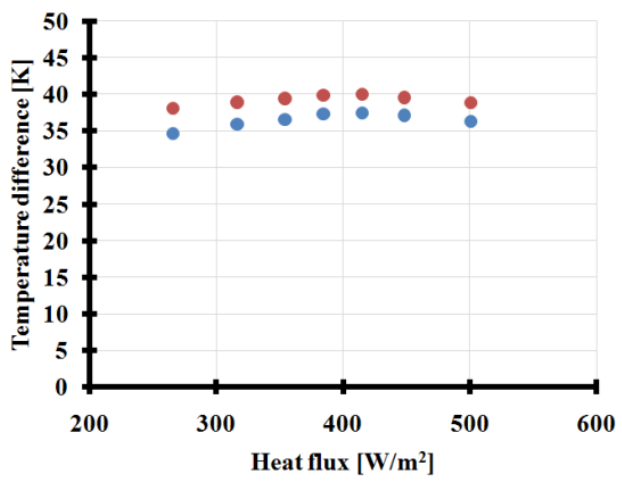

Fig. 7. Temperature differences as a function of heat flux supplied to minigap; red - evaporator, blue - condenser. 
Qualitatively, the shape of the mass flux curves is similar in both cases. For a higher heat flux, the maximum values are even smaller. On the other hand, for a smaller heat flux the periods of no flow appears, what indicates that it's not an optimal work point for this system.

For heat flux equal to $14 \mathrm{~kW} / \mathrm{m}^{2}$ a much smaller temperature gradient of vapour at the exit from the separator can be seen (less than $1.5 \mathrm{~K}$ vs $4 \mathrm{~K}$ for 7.6 $\mathrm{kW} / \mathrm{m}^{2}$ ). This can also testify the more even system operation.

\subsection{Visualisation of work of the key system components}

Because one of the walls of the evaporator is made of heat-resistant glass it is possible to register flow structures in parallel with the collection of measurement data. So, by observing the work of the evaporator it can be determined whether the given parameters are optimal or not.

The visualization of the separator's work is carried out using its special design made of plexiglass. For testing more aggressive fluids the brass version is used, as can be seen on the experimental rig diagram, Fig. 1.

The preferred way of work occurs when the boiling appears on the entire surface of the exchanger's plate. This can happen when the temperature of the working medium at the inlet to the evaporator is close to the saturation temperature. The final boiling structure at the evaporator outlet also matters because it is responsible for mass transport to the separator. For this reason, too high vapour quality is not preferred because most of the mass is transported by a less effective vapour phase.

Fig. 9. Presents a fairly favourable situation with an inlet temperature of about $10 \mathrm{~K}$ below the saturation temperature. There is a churn structure at the outlet of the evaporator what means that such a structure appears in the separator (Fig. 11.)

The situation of Fig. 10. Occurs when a bubbly structure is obtained at the outlet of the evaporator.

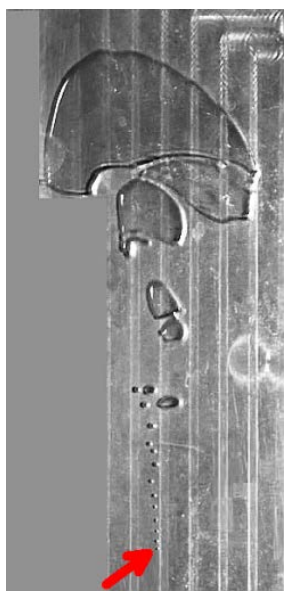

Fig. 8. Formation and growth of vapour bubbles; the arrow indicates nucleation point.

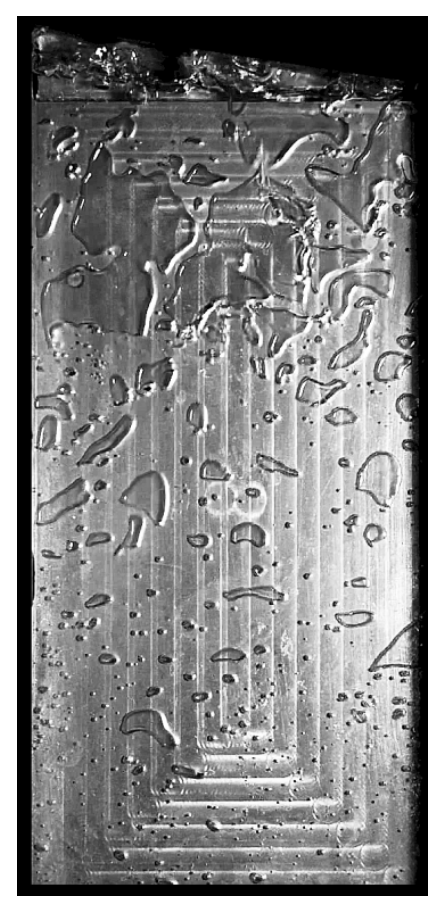

Fig. 9. The development of flow boiling on a vertically oriented plate of minigap evaporator (minigap thickness $=1$ $\mathrm{mm}$ ); visible flow structures (from the bottom to top): bubbly, slug and churn.

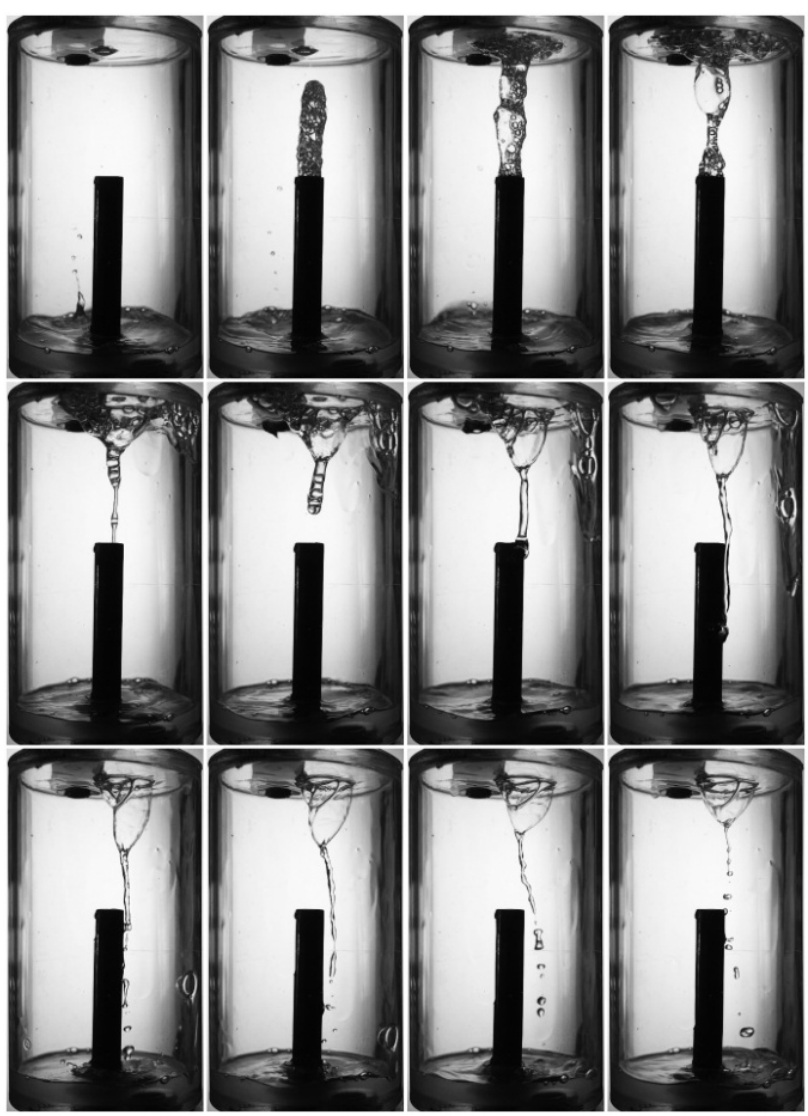

Fig. 10. Registration of single working cycle of separator ejecting boiling liquid of a bubbly structure (duration $=1 \mathrm{~s}$, time interval $=80 \mathrm{~ms}$, exposure time $=0.5 \mathrm{~ms}$ ). 


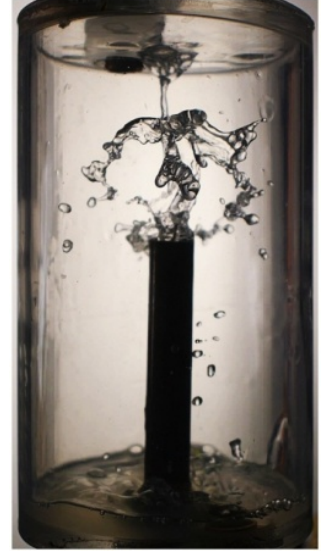

Fig. 11. Throw of a churn flow structure in separator (exposure time $=0.5 \mathrm{~ms}$ ).

\section{Experimental uncertainty analysis}

The system experimental uncertainty was determined using the sequential perturbation method of error analysis [15]. This method allows determining of the total experimental error by including errors originating from individual sources into general database and averaging it using a root sum square method (RSS). According to this method, part of measurements made using measuring instruments shows a relatively high accuracy and the resulting inaccuracies should not exceed several percent. A detailed list can be found in the Table 1 .

Table 1. Partial experimental uncertainties.

\begin{tabular}{|l|r|r|r|}
\hline \multicolumn{1}{|c|}{$\begin{array}{c}\text { Measured } \\
\text { number }\end{array}$} & $\begin{array}{c}\text { Measuring } \\
\text { range }\end{array}$ & $\begin{array}{c}\text { Absolute } \\
\text { error }\end{array}$ & $\begin{array}{c}\text { Relative } \\
\text { error }\end{array}$ \\
\hline Temperature & $90 \mathrm{~K}$ & $0.5 \mathrm{~K}$ & $0.6 \%$ \\
\hline $\begin{array}{l}\text { Evaporator } \\
\text { pressure } \\
\text { difference }\end{array}$ & $5 \mathrm{kPa}$ & $10 \mathrm{~Pa}$ & $0.2 \%$ \\
\hline System pressure & $100 \mathrm{kPa}$ & $1.8 \mathrm{kPa}$ & $1.8 \%$ \\
\hline $\begin{array}{l}\text { Mass flowrate } \\
\text { (working medium) }\end{array}$ & $5 \mathrm{~g} / \mathrm{s}$ & $0.125 \mathrm{~g} / \mathrm{s}$ & $2.5 \%$ \\
\hline $\begin{array}{l}\text { Mass flowrate } \\
\text { (heating circuit) }\end{array}$ & $8.33 \mathrm{~g} / \mathrm{s}$ & $0.172 \mathrm{~g} / \mathrm{s}$ & $2.1 \%$ \\
\hline Metric dimensions & $0.2 \mathrm{~m}$ & $0.00005 \mathrm{~m}$ & $0,025 \%$ \\
\hline Minigap thickness & $0.001 \mathrm{~m}$ & $0.00005 \mathrm{~m}$ & $5 \%$ \\
\hline
\end{tabular}

Using the above mentioned method, it was estimated that the thermal balance of the entire system was determined with an error of $5 \%$.

\section{Conclusions}

1) The system shows a cyclic way of operation, however ensuring stable way of heat transfer in the evaporator and condenser.

2) The experiment proved that a highly effective heat exchange is possible in the exchanger of a relatively small heat exchange surface. However, from the point of view of planned solar applications, it can be seen that the achieved heat flux densities are much too large. It follows, that the distilled water is not suitable as a working medium for such an applications due to the very large heat capacity. The necessity to work in a deep underpressure is also a serious disadvantage.

3) A very advantageous feature of the tested system is it's dynamic adjusting to the amount of supplied heat by changing the number of working cycles in the time, while the temperatures in exchangers remains unchanged.

\section{Acknowledgement}

The work presented in the paper was funded from the National Science Centre research project No. 2015/19/D/ST8/03201 in years 2016-2019.

\section{References}

1. P. Dąbrowski, M. Klugmann, and D. Mikielewicz, AoT, 38/3, 135 (2017)

2. A. Franco and S. Filippeschi, Exp. Therm. Fluid Sci., 51, 302 (2013)

3. M. H. Buschmann and U. Franzke, Int. J. Refrig., 40, 416 (2014)

4. J. T. Cieslinski, AoT, 37/2, 23 (2016)

5. J. Danielewicz, M. A. Sayegh, B. Śniechowska, M. Szulgowska-Zgrzywa, H. Jouhara, Energy, 77, 82 (2014)

6. L. Zhu, P. Hrnjak, Appl. Therm. Eng., 121, 879 (2017)

7. S. S. Panse, S. G. Kandlikar, Int. J. Heat Mass Transf., 106, 546 (2017)

8. R. Khodabandeh, R. Furberg, Int. J. Therm. Sci., 49/7, 1183 (2010)

9. H. Bieliński, J. Mikielewicz, AoT, 37/1, 3 (2016)

10. H. Bieliński, AoT, 37/3, 109 (2016)

11. M. Nishikawara, H. Nagano, Int. J. Therm. Sci., 102, 39 (2016)

12. A. Tamanna, P. S. Lee, Int. J. Heat Mass Transf., 89, 390 (2015)

13. T. Alam, P. S. Lee, C. R. Yap, L. Jin, Int. J. Multiph. Flow, 42, 164 (2012)

14. T. Alam, P. S. Lee, C. R. Yap, J. Heat Mass Transf., 64, 28 (2013)

15. R. J. Moffat, Exp. Therm. Fluid Sci., 1, 3 (1988) 